\title{
Substantiation of the Method of Operational Monitoring of Soil Contamination and Oil Decontamination Processes
}

\author{
Sergey Prostov ${ }^{1}$, and Evgeniy Shabanov ${ }^{1, *}$ \\ ${ }^{1}$ T.F. Gorbachev Kuzbass State Technical University, Str. Vesennyaya 28, Kemerovo, Russian \\ Federation, 650000
}

\begin{abstract}
Contamination of soils with petroleum products is an acute environmental threat. Strengthening of technogenic impact on the natural environment predetermines the need for research aimed at identifying the conditions for the safe functioning of both natural and created in the course of human industrial activity ecosystems. The method of operational determination of the degree of soil contamination with petroleum products and the use of this method for monitoring in soil purification have been studied. The method of estimation of initial and residual soil pollution is proved. The quantitative assessment of the content of oil products by the measured specific electrical resistance of the contaminated soil is investigated. The theoretical background and algorithms of software systems for assessing the content of petroleum products by using electrophysical measurements at the point, by the profile and in the volume of the soil are presented. The results of the experimental substantiation for determination of oil content in the soil sample and the comparison of the data with the results of the calculation in the software package are presented in the study. The regression dependences between the physical parameters of the soil are obtained.
\end{abstract}

\section{Introduction}

Currently, environmental issues and environmental management are becoming more urgent. Most of the problems associated with the environment arise as a result of human industrial activity. In Kuzbass, one of the most important industries is mining and processing of minerals, namely coal. Along with a large volume of industrial waste in the form of overburden dumps and the need for reclamation of disturbed lands, one of the important unsolved environmental problems of coal mining enterprises is the contamination of soils with oil products [1-3]. The problem of soil decontamination of oil products at the mining operations of Kuzbass (coal open-pit and underground mines, ore mines) is due to the large volumes of fuel consumption by road and rail, lubricants in the operation of mining equipment, technical oils in electric power plants. The main technological processes are accompanied by leakage of petroleum products into the environment. Oil refining products

\footnotetext{
* Corresponding author: shabanovea@kuzstu.ru
} 
contain toxic water-soluble fractions, in addition, gasoline and especially motor oils contain up to $20 \%$ of additives, solvents, heavy metals [4-5].

To substantiate the most effective parameters of the technology of soil purification from the pollutant, the initial and residual pollution are evaluated [6-7]. Modern methods of assessing the content of pollutants in the soil can be very time-consuming, often associated with significant losses of time spent on the preparation and laboratory testing of soil samples [8-9]. Operational monitoring of the state, properties of soils and physical and chemical processes in the electrically treated area can be provided by geophysical methods, which are an effective complement to engineering-geological surveys. Electrophysical and electromagnetic methods based on the measurement of the parameters of electric fields are very promising for the study of oil-contaminated soils, since oil and petroleum products exhibit pronounced dielectric properties and are therefore electrically contrasting. KuzSTU has been developing a method of rapid determination of the content of oil products in the soil for several years $[10]$.

\section{Method of research}

The basic physical premise of the control method is that all petroleum products used in road and rail transport (oils, diesel fuel, gasoline) exhibit pronounced dielectric properties. The effective resistivity of these fluids varies in the range $\rho=10^{10}-10^{12} \mathrm{Ohm} \cdot \mathrm{m}$, while for natural mineral solutions this range is $\rho=0.1-100 \mathrm{OM}^{*} \mathrm{M}$. Specific electrical resistance of watersaturated rock, which is a three-phase medium, is determined by the classical empirical dependence:

$$
\rho_{k}=m^{-\beta} W^{-\gamma} \rho_{v}
$$

where $m$ - porosity (pore emptiness); $W$ - the ratio of the saturation space of the pores and cracks; $\rho_{v}-$ the resistivity of the solution, filling pore space, Ohm.m; $\beta, \gamma$ - empirical parameters depending on the structural and textural features of the studied soils (rocks); $\beta$ parameter determined by the structure of the pore space (mainly the tortuosity of the channels); $\gamma$-parameter depending on the wettability of the pore surface solution.

The value of parameter $\beta$ depends on the structure of the pore space and for compacted non-cohesive soils it varies in the range $\beta=1.3-2.2$; parameter $\gamma$ is determined by the degree of wettability of the surface of the solid phase and it varies in the range $\gamma=1.8-3.5$.

The value ranges of parameter $\beta$ and $\gamma$ are obtained by inverse calculations using a bank of experimental data $\rho_{k} / \rho_{v}$, as well as the average values of physical and technical parameters $m$ and $W$ for the main types of clay soils of Kuzbass [11].

Table 1. The predicted values of the structural parameters $\beta$ and $\gamma$.

\begin{tabular}{|c|c|c|c|c|c|c|c|}
\hline \multicolumn{2}{|c|}{ Type of soil } & Parameter & \multicolumn{5}{|c|}{$\boldsymbol{\rho}_{k} / \mathbf{\rho}_{\mathbf{v}}$} \\
\cline { 3 - 8 } & & $\gamma$ & 10.0 & 5.0 & 2.0 & 1.5 & 1.2 \\
\hline \multirow{4}{*}{$\begin{array}{c}\text { Sandy loam } \\
\text { Parameter }\end{array}$} & 1.8 & 0.59 & 1.18 & 1.96 & 2.20 & 2.40 \\
\cline { 3 - 8 }$\beta$ & $W=0.314 ;$ & 2.2 & 1.15 & 1.74 & 2.51 & 2.77 & 2.95 \\
\cline { 3 - 8 } & $W=0.187)$ & 2.6 & 1.72 & 2.32 & 3.13 & 3.34 & 3.53 \\
\cline { 3 - 8 } & Loams & 1.8 & 0.30 & 0.87 & 1.62 & 1.69 & 2.04 \\
\cline { 3 - 8 } & $(m=0.296 ;$ & 2.2 & 0.79 & 1.36 & 2.11 & 2.35 & 2.53 \\
\cline { 3 - 8 } & $W=0.227)$ & 2.6 & 1.27 & 1.84 & 2.60 & 2.83 & 3.02 \\
\cline { 3 - 8 } & Clays & 1.8 & 0.62 & 1.23 & 2.05 & 2.30 & 2.50 \\
\cline { 3 - 8 } & $(m=0.324 ;$ & 2.2 & 1.21 & 1.82 & 2.64 & 2.89 & 3.09 \\
\cline { 3 - 8 } & $W=0.189)$ & 2.6 & 1.80 & 2.41 & 3.23 & 3.84 & 4.46 \\
\hline
\end{tabular}

To quickly determine the pollutant content in the pore liquid, it is advisable to use the 
theoretical dependence of the harmonic weighted average for a two-component medium, which will allow us to calculate the desired value with great reliability:

$$
\rho_{v}{ }^{-1}=\sum_{i=1}^{n} V_{i} \cdot \rho_{i}^{-1}=V_{e} \cdot \rho_{e}^{-1}+V_{n} \cdot \rho_{n}^{-1}
$$

where $\rho_{v}, \rho_{n}, \rho_{e}$ - specific electrical resistance of the weighted average, oil product and electrolyte, respectively, Ohm·m; $V_{n}, V_{e}$ - the volume of the solution, respectively of the oil and of the electrolyte.

Theoretical dependences (1) and (2) allow us to quickly determine the degree of soil contamination by oil products based on the results of single measurements, longitudinal and areal electrical probes.

\section{Results and Discussion}

For further analysis of the process of electrochemical treatment of contaminated soils, the information about the electrophysical properties of natural aqueous solutions and liquids containing pollutants and saturating soils was required. The experimental studies of the electrical properties of electrolyte solutions containing petroleum products were performed with DC and AC measurements.

The results of specific electrical resistivity measurements $\rho_{e}$ of $\mathrm{NaCl}$ solution in relation to salt concentration in the solution, AC frequency, and similar dependences of specific electrical resistance $\rho_{n}$ of motor oils and gasoline have shown that with the change in the concentration of the aqueous salt solution up to $6.5 \%$, the resistivity decreases from 80 до $0.2 \mathrm{Ohm} \cdot \mathrm{m}$, and the specific electrical resistance of motor oils, gasoline and solvent in the frequency range $f=0-1 \mathrm{kHz}$ varies in the range of $(0.2-1.1) \cdot 10^{6} \mathrm{Ohm} \cdot \mathrm{m}$.

From the above data it follows that all petroleum products are electrically very contrast, because their resistivity is at least by 3 orders higher than the resistivity of natural aqueous solutions.

By substituting these data into equation (2), we obtain the dependences of the mean values $\rho_{v}$ on the relative content of petroleum products $V_{\mathrm{n}} / V_{\mathrm{e}}$ for DC (Fig. 1) and AC (Fig. 2).

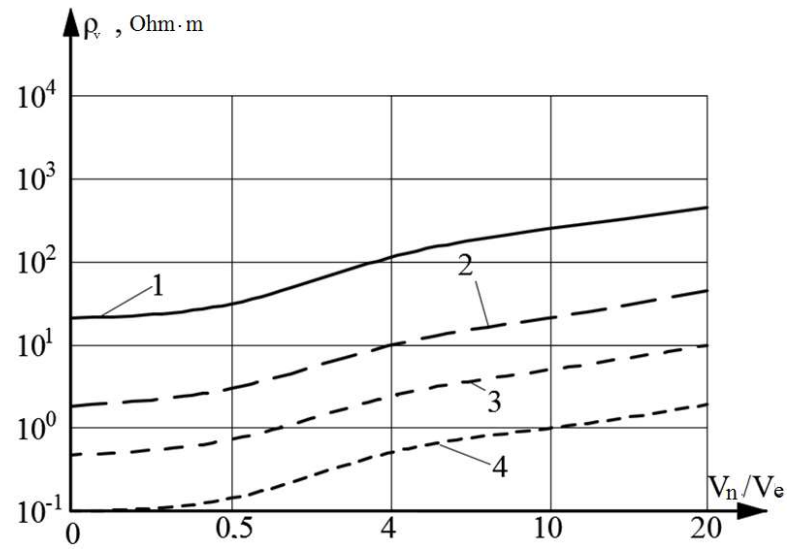

Fig. 1. Dependence of mean values $\rho_{v}$ on the relative content of petroleum products when measured at DC current: 1 - salt concentration in solution $C=0.1 \mathrm{~g} / 1 ; 2-C=0.5 \mathrm{~g} / 1 ; 3-C=2 \mathrm{~g} / 1 ; 4-C=10 \mathrm{~g} / 1$. 

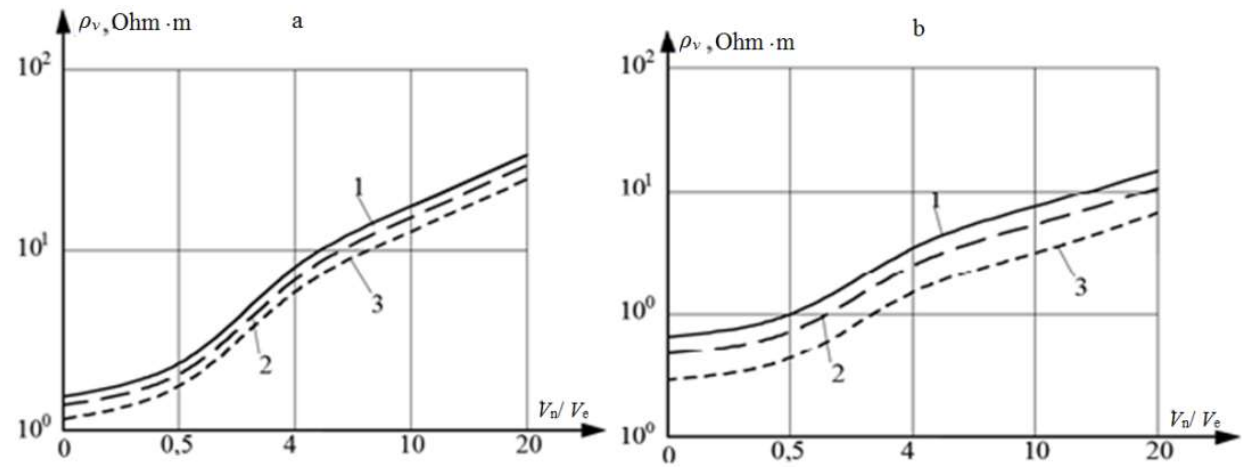

Fig. 2. Dependence of the average resistivity on $\mathrm{AC}$ frequency and concentration of salt in the electrolyte $C=0.5 \mathrm{~g} / 1$ (a); $10 \mathrm{~g} / \mathrm{l}(\mathrm{b}): 1-f=10 \mathrm{~Hz} ; 2-200 \mathrm{~Hz} ; 3-1000 \mathrm{~Hz}$.

Taking into account the ranges of structural parameters $\beta$ and $\gamma$ shown in Table 1, we have calculated the dependences of changes in the effective resistivity of main clay soils of the quaternary deposits of Kuzbass, partially presented in Figure 3.
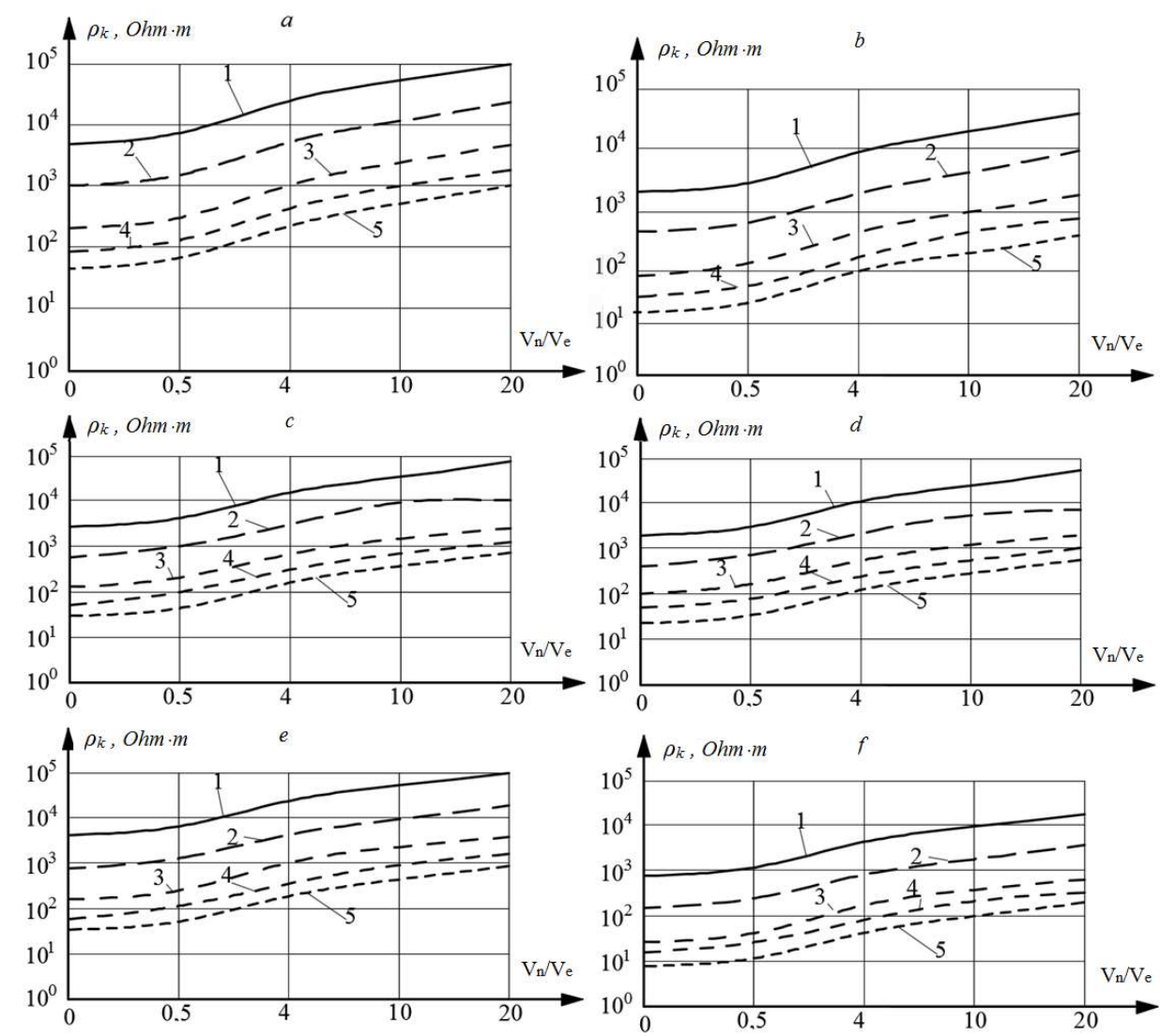

Fig. 3. Dependences of the effective resistivity $\rho_{k}$ of Kuzbass soils on the coefficient of moisture saturation of the pore space and cracks $\mathrm{W}$, the relative content of oil in the weighted average $V_{\mathrm{n}} / V_{\mathrm{e}}$ and the salt concentration in the electrolyte $\mathrm{C}, \mathrm{g} / \mathrm{l}$ : sandy loam $-C=0.5$ (a), $C=10$ (b); loams' $-C=0.5$ (c), $C=10$ (d); clays' - $C=0.5$ (e), $C=10$ (f): $1-W=0.1 ; 2-W=0.2 ; 3-W=0.4 ; 4-W=0.6 ; 5-W=$ 0.8 . 
These dependences allowed us to form the database for the interpretation of the results of experimental probes in the control of changes in the ratio $V_{\mathrm{n}} / V_{\mathrm{e}}$ in size $\rho_{k}$.

To quantify the degree of pollutant saturation, the soil pollution coefficient $k$, was introduced equal to the ratio of the volume of oil contained in the pores of the soil to the total volume of pore fluid. By transforming equations (1) and (2), the following expression can be obtained:

$$
V_{n}=\frac{\rho_{n}-\frac{\rho_{e} \rho_{n}}{\rho_{k} m^{\beta} W^{\gamma}}}{\rho_{n}-\rho_{e}} \approx 1-\frac{\rho_{e}}{\rho_{k} m^{\beta} W^{\gamma}},
$$

where $V_{n}$ - the volume of the pores filling with oil;

$$
k=m \cdot V_{n},
$$

where $k$ - the contamination factor.

The theoretical dependence (3) allows us to estimate the degree of soil contamination by oil products on the basis of the results of single measurements, profile and area electric probes using cyclic algorithms, which are implemented in a set of programs created in the software product Lazarus (FreePascal).

Figure 4 shows the algorithm (block diagram) for calculating the local soil pollution index.
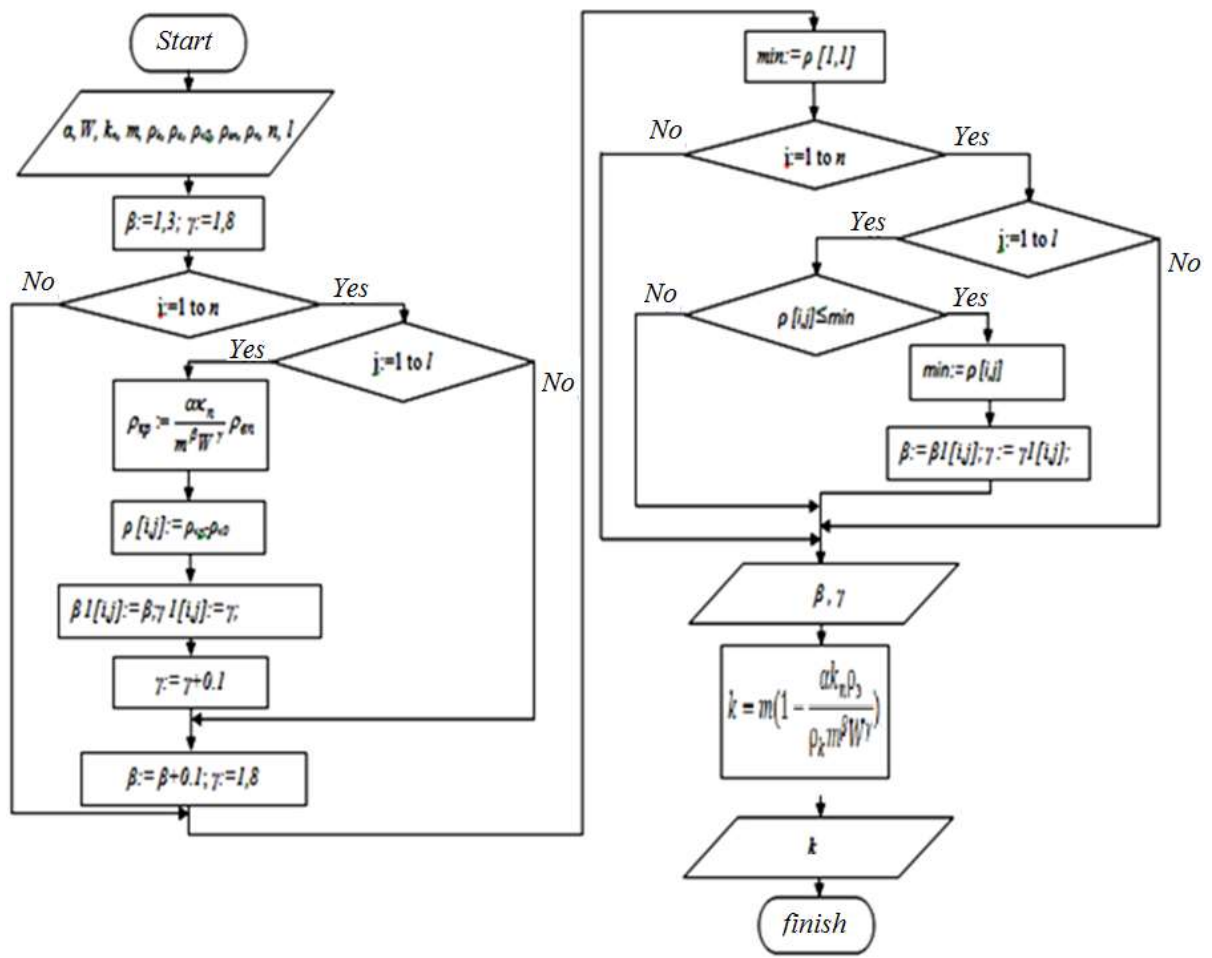

Fig. 4. The block diagram for calculation of local coefficient of soil contamination with oil products: $\rho_{v}, \rho_{k} 0, \rho_{n}$ - resistivity of the electrolyte, the ground outside the contaminated zone, and oil, respectively, $\mathrm{Ohm} \cdot \mathrm{m} ; n$ and $l$ - specified number of search cycles for optimal values $\beta$ and $\gamma ; k$ - the contamination factor of soil.

The algorithm can be divided into several blocks. In the first block, the measured resistivity of sample fluid $\rho_{v n}$, dependence (1), the reference data, and the results of the 
preliminary experiment are used to determine the optimal values of the structural parameters $\beta$ and $\gamma$, at which the values obtained by calculation differ in minimum from the experimental ones. The second block is responsible for the calculation of the degree of soil pollution on the basis of the results obtained and using the dependence (3).

The proposed method allows us to:

- promptly assess the degree of soil contamination with oil or oil products;

- substantiate recommendations for decision-making on the choice of optimal parameters of oil-contaminated soil treatment technology and remediation of the natural environment.

For an average assessment of the degree of soil contamination by profile (section) or volume it is advisable to use an integral indicator, including the bank of local values $k_{i}$ and areas $S_{i}$ (volumes $V_{i}$ ) of contaminated areas:

$$
I_{S}=\frac{\sum k_{i} \cdot S_{i}}{S}, \quad I_{V}=\frac{\sum k_{i} \cdot V_{i}}{V} .
$$

The block diagram for the calculation of integral indicators $I_{s}, I_{V}$ is shown in Figure 5.

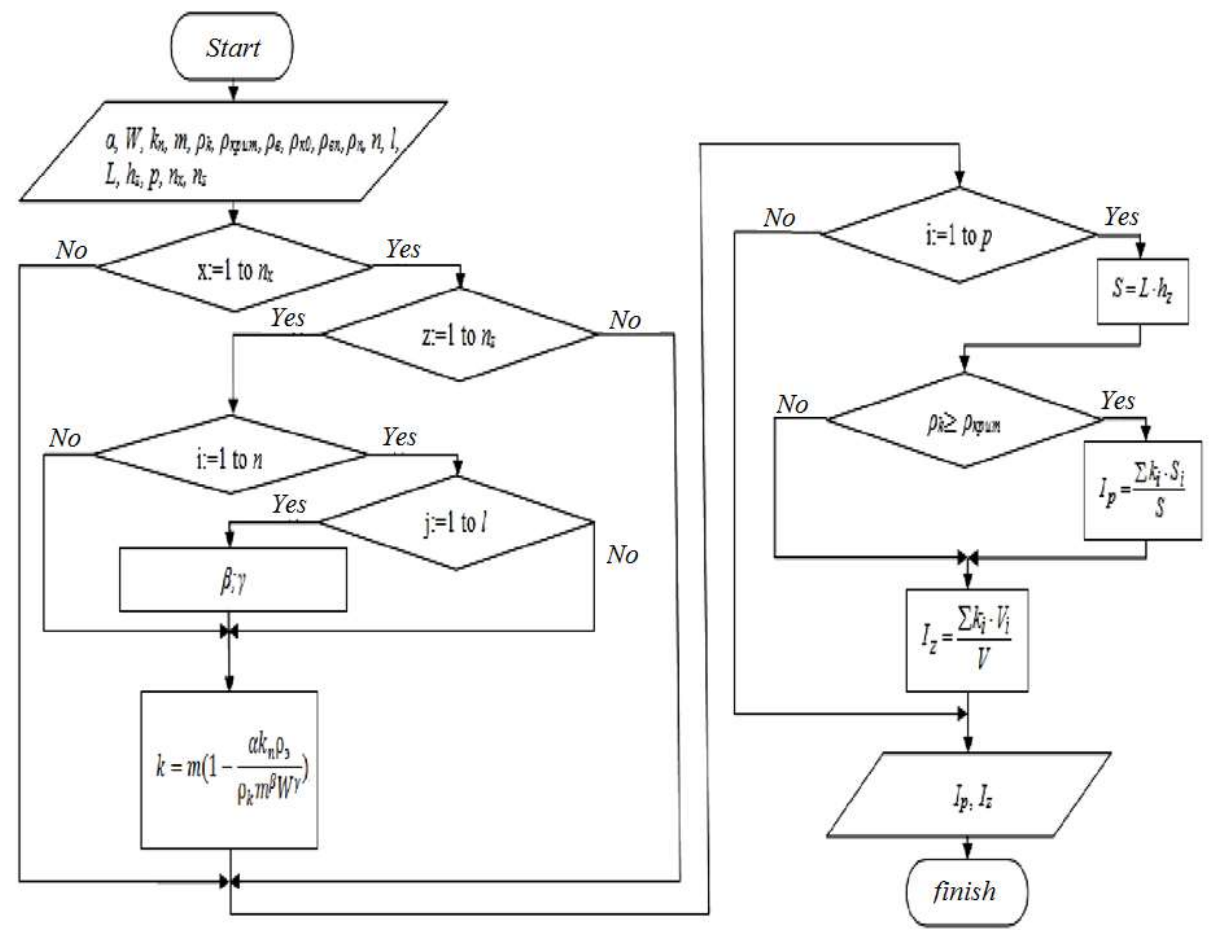

Fig. 5. Block diagram for calculation of the integral index of soil pollution by oil products on the profile $I_{S}$ and by volume $I_{v}: L$ - profile length, $\mathrm{m} ; h_{z}$ - sensing depth, $\mathrm{m} ; S$ - area of the measured profile, $S=L \cdot h_{z}, \mathrm{~m}^{\wedge} 2 ; p-$ number of control profiles; $\rho_{k r}$ - critical value of resistivity in the profile, Ohm $\cdot \mathrm{m} ; n_{x}$ - number of measurement points along the length of the profile; $n_{z}$ - number of measuring points for depth of profile 6.

The geometric parameters of the pollution zone used in the algorithm are shown in Figure 


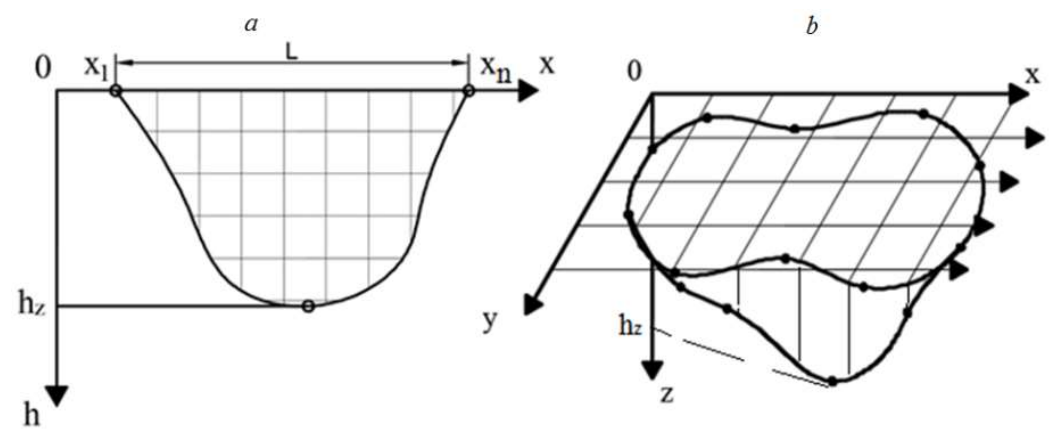

Fig. 6. Geometrical parameters of the pollution zone used for calculation of integral indicators $I_{\mathrm{s}}(\mathrm{a})$ and $I_{\mathrm{v}}(\mathrm{b})$

To check the theoretical dependences used in the calculation of the pollution coefficient $k$, an experiment was conducted on samples of artificially contaminated soils.

Samples of contaminated soil were obtained by mixing clean soil with a fixed amount of engine oil using a mixer. For artificially contaminated soil samples in the experiment, we determined the porosity, moisture content, resistivity of soil $\rho_{k}$, electrolyte $\rho_{\ni}$ and pore liquid $\rho_{H}$, as well as the coefficient of soil pollution $k$ with the help of a specially developed software package that provides a cyclic selection of optimal values of constants $\beta$ and $\gamma$.

During the experiment with artificially contaminated samples, starting with sample \#7, a partial violation of the structural bonds of the sample was observed. With an increase in the concentration of petroleum $k>30 \%$ there was a change in the phase state of the sample: its transition from a solid cohesive to a plastic disjoint suspension, which contributed to a sharp increase in the resistivity of the sample $\left(\rho_{k} \rightarrow \rho_{n}\right)$. The scope of the proposed method of control is limited by $k<20 \%$, in this case, the error of evaluation does not exceed $23 \%$ (Table 2).

Table 2. Physical characteristics of soil in experimental samples (mass of soil $m_{g}=200 \mathrm{~g}$, mass of water $m_{w}=20 \mathrm{~g}$, humidity $W=22 \%$ ).

\begin{tabular}{|c|c|c|c|c|c|}
\hline \multicolumn{2}{|c|}{ Characteristics } & $\begin{array}{c}\text { Oil weight, } \\
\mathrm{g}\end{array}$ & $\begin{array}{c}\text { Specific electrical } \\
\text { resistance of soil, } \\
\rho k, \text { Ohm } \cdot \mathrm{m}\end{array}$ & $\begin{array}{c}\text { Porosity, } \\
m, \%\end{array}$ & $k, \%$ \\
\hline \multirow{5}{*}{} & 1 & 0 & 13.75 & 38.8 & 0 \\
\cline { 2 - 6 } & 2 & 2 & 17.5 & 40.7 & 1.0 \\
\cline { 2 - 6 } & 3 & 4 & 21.25 & 42.5 & 1.8 \\
\cline { 2 - 6 } & 4 & 10 & 23.75 & 48.1 & 4.35 \\
\cline { 2 - 6 } & 5 & 15 & 22.5 & 52.7 & 6.4 \\
\cline { 2 - 6 } & 6 & 20 & 25.0 & 57.4 & 8.3 \\
\cline { 2 - 6 } & 7 & 30 & 26.25 & 66.7 & 12.0 \\
\cline { 2 - 6 } & 8 & 40 & 42.5 & 76.0 & 15.4 \\
\cline { 2 - 6 } & 9 & 50 & 950.0 & 85.3 & 18.5 \\
\hline
\end{tabular}

For geophysical express-forecast it is possible to choose a regression dependence, which accurately describes the nature of the change in pollution coefficient $k$ as the concentration of petroleum products in the soil increases and the corresponding relative increase in specific electric resistance $\rho_{\kappa}$. Figure 8 shows the result of the aggregation of the regression equation and related parameters: correlation ratio (correlation coefficient) $R$; Fisher criterion, $F$; critical value of Fisher criterion, $F_{\mathrm{kr}}$. The polynomial dependence provides the highest accuracy $(R=0.981)$. 


$$
k=18.57\left(\frac{\rho_{k}}{\rho_{k 0}}\right)^{2}-42.7257 \frac{\rho_{k}}{\rho_{k 0}}+24.44
$$

herewith: $\mathrm{R}=0.981, \mathrm{~F}_{\mathrm{kr}}=9.552, \mathrm{~F}=81.746$
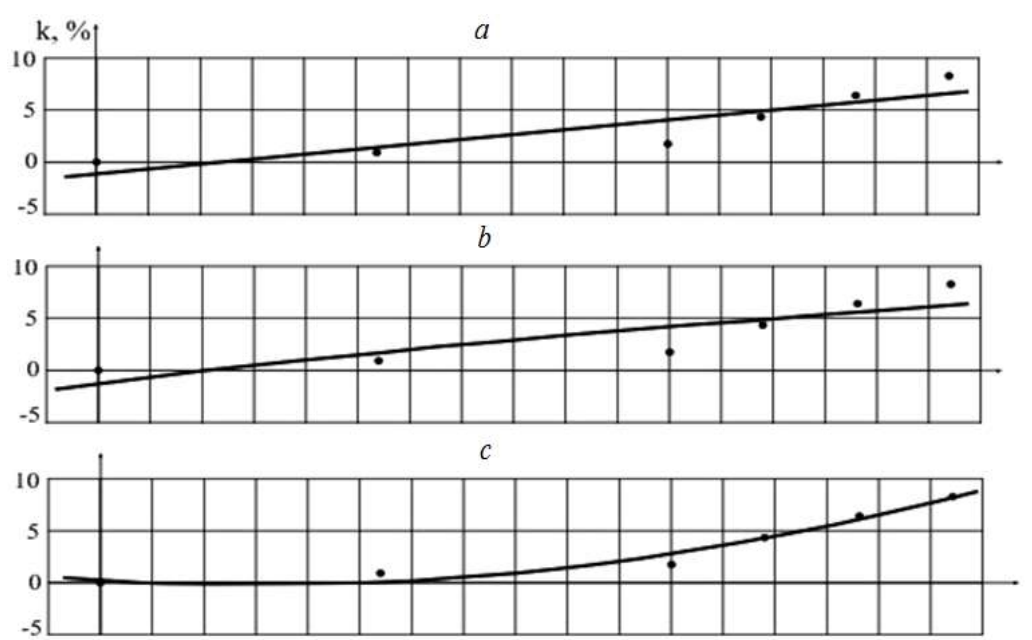

$d$

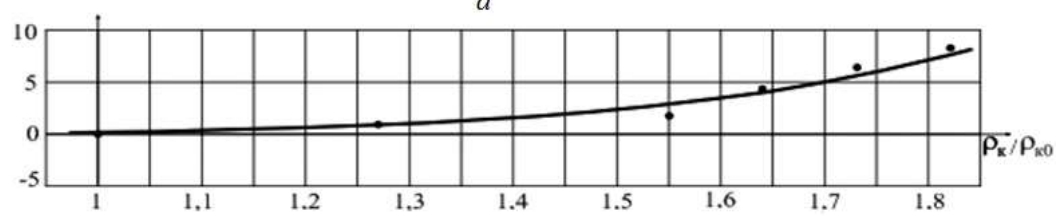

Fig. 7. Results of statistical processing of the experimental dependence of the pollution coefficient on the ratio of electrical resistances $\rho_{\mathrm{k}} / \rho_{\mathrm{k} 0}$ of soil in the sample; linear (a); logarithmic (b); polynomial (c); power-law (d).

The program calculation allowed us to estimate the changes of the structural parameter $\beta$ as the concentration of the pollutant in the soil increased (Fig. 8). It follows from the graph that for the region corresponding to the phase transition $\left(\rho_{\mathrm{k}} / \rho_{\mathrm{e}}>190\right)$, the values of the structural indicator $\beta$ should be further adjusted in relation to the values given in Table.1.

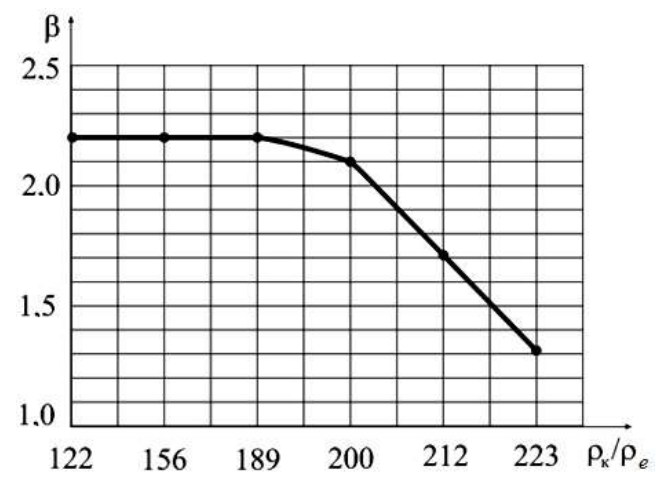

Fig. 8. The dependence of the structural index $\beta$ on the relative resistivity of the soil in the sample.

The results of the laboratory experiment allowed us to establish the following:

- the method of electrical resistance is effective for rapid assessment of the degree of 
contamination of the soil with petroleum products at their content up to $15-20 \%$, after which the destruction of the structural bonds of the soil takes place;

- to calculate the pollution coefficient $k$, it is advisable to use the classical empirical dependences of electrical exploration for the resistivity of a three-phase medium, implemented in the form of cyclic algorithms by optimizing the basic structural parameters and adjusting them for the phase transition region;

- polynomial dependence of $k$ on the relative resistivity is the most suitable for an approximate assessment of the degree of pollution.

\section{Conclusion}

The theoretical and experimental studies on the justification of methods of electrophysical control of the degree of contamination of soils with petroleum products allowed us to draw the following conclusions.

To control the degree of soil contamination with petroleum products, it is advisable to use the dependence of the resistivity of the three-phase medium on porosity, humidity and resistivity of the pore filler which is used in electrical exploration and includes structural and textural empirical parameters. In this case, the ranges of parameters of the structure of the pore space $\beta=1.3-2.2$ and wettability $\gamma=1.8-3.3$ should be taken for the conditions of Kuzbass in accordance with previously established experimental data, and the resistivity of the pore filler should be calculated by the formula of a harmonic weighted average for a twocomponent medium.

It is experimentally established that with a change in the concentration of an aqueous solution of salt to $6.5 \%$, the specific electrical resistance decreases from 80 to $0.2 \mathrm{Ohm} \cdot \mathrm{m}$, and the specific resistivity of engine oil and gasoline in the frequency range $\mathrm{f}=0-1 \mathrm{kHz}$ ranges $(0.2-1.1) \cdot 10^{\wedge} 6 \mathrm{Ohm} \cdot \mathrm{m}$. Using the experimental and theoretical dependences, we obtained database resistivity of pore filler and an effective resistivity of three-component medium for the specified ranges of concentrations of the aqueous solutions of the frequencies and the ratio of volume fractions of oil and of the electrolyte $V_{\mathrm{n}} / V_{\mathrm{v}}=0-20$.

To make the calculation of the local soil pollution coefficient $\mathrm{k}$ based on the experimental data of electrical probes automatic, an algorithm has been developed that includes the selection of optimal values of structural parameters $\beta$ and $\gamma$ according to the criterion of the minimum deviation of the calculated value of the effective resistivity from the measured one and the calculation of values $\mathrm{k}$ in relation to the values of the effective resistivity within the pollution zone and outside it.

To improve the accuracy of estimation of the degree of contamination of the proposed integral parameters $I_{s}$ and $I_{v}$, including the summation of the products of local values $k$ and elementary areas or volumes into which the pollution zone is divided at a given step.

For the experimental verification of the accuracy of the calculated dependences and the computer program, a comparison of the soil pollution coefficients was carried out, with which a fixed volume of waste oil was mixed at a known humidity and porosity. The experiment showed that in the range $k<20 \%$ the estimation error did not exceed $23 \%$. At $k>30 \%$, the soil sample passed from a cohesive state to a plastic disjoint suspension, as a result of which the magnitude of $\rho_{\mathrm{\kappa}}$ and prediction error increased sharply. It was also found that to improve the accuracy of the calculation, the value of the structural parameter $\beta$ should be adjusted in accordance with the established nonlinear dependence at $\rho_{\mathrm{k}} / \rho_{\mathrm{e}}>190$.

For the express analysis in field studies, one can use regression of the dependences of $k$ on the relative value $\rho_{\mathrm{k}} / \rho_{\mathrm{k} 0}$, while polynomial, power and linear dependences have correlation ratio $\mathrm{R}>0.9$. 


\section{References}

1. B. Amina, B. Djamel, S. Sofiane, S. Ladjel, AIP Conference Proceedings, 145, 1758, 030040 (2016)

2. Terry J. McGenity, Current Opinion in Biotechnology, 27, 46 (2014)

3. B. Veronica, C. Brunello, Journal Toxicological \& Environmental Chemistry, 92, 45 (2010)

4. A. Boukharov, A. Balashov, A. Timohin, A. Ivanov, B. Holin, J. Ph., 2, 012131 (2017)

5. T. E. Litvinova, M. A. Sulimova, O. V. Cheremisina, Mining Ecology Management, 34, 1017 (2017)

6. N. V. Morozov, I. M. Ganiev, Research journal of pharmaceutical, biological and chemical sciences, 7:5, 1728 (2016)

7. E. I. Krapivsky, V. O. Nekuchaev, A. E. Beljaev, A. D. Charnetsky, 67th European Association of Geoscientists and Engineers, 1, 2753 (2005)

8. S. W. Park, K. J. Kim, K. Baek, J. Y. Lee, J. S. Yang, Separation Science and Technology, 45:12, 1988 (2010)

9. C. J. Tsai, C. H. Feng, J. H. LI, Journal of chromatographya, 1410, 60 (2015)

10. Prostov S. M., Shabanov E. A. Proceedings of the 8th Russian-Chinese Symposium, 92, 175 (2016)

11. S. M. Prostov, E. A. Shabanov, E3S Web Conf., 21, 02007 (2017) 